\title{
Dorsoventral patterning in plants
}

\author{
Allen Sessions $^{1}$ and Martin F. Yanofsky ${ }^{1}$ \\ Department of Biology, University of California at San Diego, La Jolla, California 92093-0116 USA
}

Although mutations affecting dorsoventral patterning in plants have been known for centuries (Linnaeus 1749), the underlying mechanisms controlling this process have remained mysterious. Recent papers by Sawa et al. (1999b) and others have begun to shed light on this subject through the isolation and characterization of genes responsible for dorsoventrality. In plants, lateral organs and shoots are initiated from the flanks of the shoot apical meristem (SAM), a multilayered dome of self-organizing and self-perpetuating cells. Primordia emerge from the SAM with a characteristic asymmetry and identity that depend on both extrinsic and intrinsic factors (Fig. 1). For leaf primordia, this means having distinct upper and lower programs of growth and cellular differentiation so that cells in the upper half develop the appropriate epidermis, palisade mesophyll, and xylem strand, and cells in the bottom half develop a phloem strand, a spongy mesophyll, and appropriate epidermis. Organ shape and cell-type differences between the adaxial (upper) and abaxial (lower) sides of the mature leaf ensure that the plant puts the bulk of its light-gathering capacity toward the light.

The asymmetry in the shape of most leaves is apparent from inception as they emerge as a crescent on the flank of the SAM, and this dorsoventrality is further accentuated by differential growth of the adaxial and abaxial sides. Early asymmetry is also revealed by adaxial- and abaxial-specific expression of genes in incipient and young organ primordia (Fig. 1; see also Sawa et al. 1996). Dorsoventral patterning also occurs in lateral primordia that give rise to asymmetric shoots, such as the snapdragon flower, in which the upper and lower positioned petals and stamens differ. The dorsoventrality of this and other asymmetric lateral shoots is a result of the differences in the initiation and growth of organs on the adaxial and abaxial sides of the lateral SAM, and also to the asymmetry of the lateral SAM itself.

\section{How do flies do it?}

The problem of dorsoventral patterning of appendages is best understood in the developing Drosophila wing (for review, see Brook et al. 1996; Lawrence and Struhl 1996).

\footnotetext{
${ }^{1}$ Corresponding author.

E-MAIL asession@biomail.ucsd.edu; marty@ucsd.edu; FAX (619) 822 1772.
}

The process begins as the wing imaginal disc forms as an invagination of the embryonic ectoderm (for review, see Cohen 1993). The wing disc is then subdivided into dorsal and ventral lineage compartments. The primary gene involved in specifying dorsal cell identities is apterous $(a p)$, which is expressed in dorsal but not ventral cells (Cohen et al. 1992; Diaz-Benjumea and Cohen 1993; Blair et al. 1994). ap then specifies dorsal identity and directs the expression of a number of other genes leading to activation of wingless ( $w g$ ) expression along the dorsoventral boundary (Irvine and Wieschaus 1994; DiazBenjumea and Cohen 1995; Kim et al. 1995; Rulifson and Blair 1995; DeCelis et al. 1996; Neuman and Cohen 1996). Wg is a secreted protein that acts as a morphogen to specify dorsal and ventral cell fates in the developing wing (Zecca et al. 1996; Neuman and Cohen 1997). The juxtaposition of dorsal and ventral cells induces the expression of $w g$ and the formation of the organizing center at the margin, resulting in outgrowth of the wing (Blair et al. 1994; Williams et al. 1994).

From these and other studies it has been proposed that many aspects of patterning in developing animals could be the result of a similar developmental paradigm, beginning with the establishment of domains or compartments. Subsequently, short-range signaling across the boundary of the two domains ultimately leads to the production of a diffusible long-range signaling molecule that specifies pattern as a morphogen from the boundary position (Lawrence and Struhl 1996).

\section{Classical studies on dorsoventral patterning in plants}

Early work pointed to the importance of the SAM in supplying signals to newly initiated primordia on its flanks. Surgical incisions that separated incipient leaf primordia from the parent SAM resulted in their development as radialized leaves, or centric organs, that lacked distinct adaxial and abaxial identities (Sussex 1955; Snow and Snow 1959; Hanawa 1961). These and other results led to the proposal that the SAM sent a growth-inhibiting signal to the adaxial surface of leaf primordia, leading to asymmetric growth and the initiation of dorsoventrality (Wardlaw 1949). However, other experiments documenting the development of dorsoventral leaves in the absence of a SAM do not support this hypothesis (discussed in Steeves and Sussex 1989). One report suggested that it was the asymmetric influence of gravity that was responsible for dorsoventral patterning 


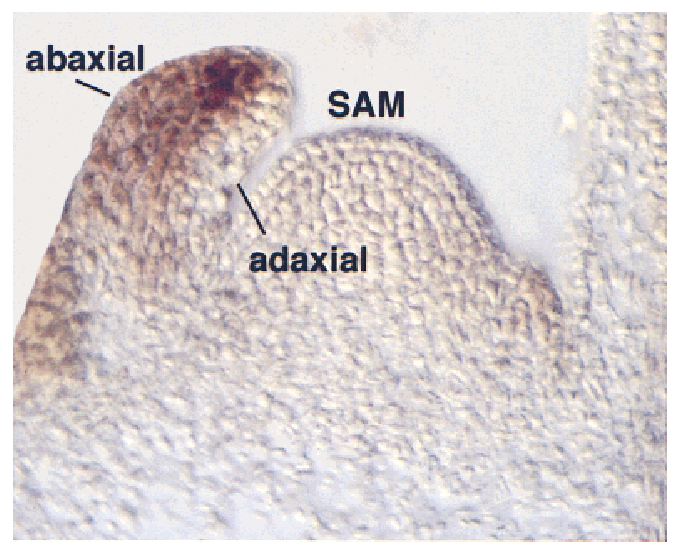

Figure 1. Longitudinal section of an Arabidopsis shoot apex showing the SAM and a young leaf primordium with abaxial and adaxial sides indicated, and showing expression of FIL (brown stain) on the abaxial side of a young leaf primordium (image kindly provided by John Bowman). The early literature defines abaxial as dorsal, whereas more recent usage in the literature refers to the adaxial surface as dorsal. To avoid confusion, in this review we use the terms abaxial and adaxial.

in some flowers, as plants with normally asymmetric flowers formed symmetric flowers when grown in a clinostat (Vöchting 1884).

\section{Asymmetrically expressed factors}

More recent insights to dorsoventral patterning in plants have come, like they did with flies, in the form of mutants that have developmental phenotypes that cause the formation of adaxial tissues in place of abaxial and vice versa. The first clues came with the characterization of the CYCLOIDEA (CYC) and DICHOTOMA (DICH) genes from snapdragon (Luo et al. 1996). Snapdragon is similar to many plants that have asymmetric flowers, and cyc dich double mutants produce abaxialized flowers that are radially symmetric (Coen 1996; Luo et al. 1996). CYC and DICH encode closely related proteins, consistent with genetic data suggesting redundancy, and are similar to another regulator of lateral outgrowth in plants, the maize TEOSINTE BRANCHED1 (TB1) product (Doebley et al. 1997). The homology between CYC and TB1 includes a 62-amino-acid region containing a putative nuclear localization signal, suggesting that these proteins may function as transcriptional regulators (Doebley et al. 1997). CYC is expressed in the adaxial half of the floral primordium, and in adaxially positioned floral organ primordia, where it controls growth rate and primordia initiation (Luo et al. 1996). The development of abaxial identities in place of adaxial in cyc dich double mutants suggests that $C Y C / D I C H$ act to repress the development of abaxial identities in the adaxial side of the floral primordium. Interestingly, the activity of the DIVARICATA gene product from snapdragon appears to be required for abaxial identity and is restricted to the abaxial part of the primordium by an antagonistic activity of CYC and DICH (Almeida et al. 1997).

\section{Symmetrically expressed factors}

Clues to dorsoventral patterning during leaf development came with the isolation of the snapdragon PHANTASTICA (PHAN) gene (Waites et al. 1988). phan mutants have needle-shaped abaxialized leaves and petals that largely lack adaxial structures and laminae (Waites and Hudson 1995). Early leaves have patches of abaxial tissue on the adaxial surface, and where the two cell types meet ectopic leaf margins form. The abaxialized needle leaves that lack laminae, as well as the ectopic laminae that surround patches of abaxial cells on the adaxial surface of the early leaves in phan mutants, both strikingly suggest that the juxtaposition of adaxial and abaxial identities can lead to the induction of lamina outgrowth. Temperature-shift experiments show that PHAN is only needed for dorsoventrality before primordium emergence, suggesting that this is specified in the SAM as suggested by earlier surgical experiments (Waites et al. 1988). PHAN encodes a MYB-related transcription factor that is expressed in all cells of all lateral organ primordia initiated from shoot and floral meristems (Waites et al. 1988). Because the abaxialized phenotypes occur in only a subset of lateral organs of phan mutants, the observed expression pattern implies that other factors modulate PHAN activity.

\section{YABBY genes promote abaxial cell fate?}

Now, articles by Sawa et al. (1999b), and Bowman and Smyth (1999) report the existence of a gene family that potentially controls abaxial patterning in Arabidopsis. The paper by Sawa et al. in this issue describes the isolation and expression pattern of the FILAMENTOUS FLOWER (FIL) gene (Sawa et al. 1999b). fil mutants have pleiotropic defects in the initiation and development of both floral meristems and floral organs (Sawa et al. 1999a). fil mutants produce two types of aberrant flowers that are arranged in three zones along the infloresence stem. The first 10-15 flowers produced have misshapen, aberrantly positioned, or missing floral organs. This is followed by the production of 20 nodes of filament structures, and finally by a zone of both types of structures. The filament-like structures appear to be partial flowers composed only of the basal pedicel, and lacking the distal receptacle, or organ-bearing part of the flower (Sawa et al. 1999a). The common feature among the two types of aberrant flowers is the lack of a full complement of floral organs.

fil single mutant phenotypes may provide only a partial picture of FIL function, however, as FIL is a member of a multigene family in Arabidopsis that includes closely related members. Clues to the function of FIL not apparent from the fil single-mutant phenotype come from expression studies showing that FIL RNA normally accumulates only on the abaxial side of leaves, floral meristems, and all floral organs (Sawa et al. 1999b). Moreover, when the FIL gene is expressed ectopically, the leaves either develop as arrested cylindrical structures that fail to initiate lamina outgrowth, or as 
wrinkled organs that develop patches of abaxial epidermal cells on the adaxial surface (Sawa et al. 1999b). Taken together, these results suggest that the normal role of FIL may include promotion of abaxial cell fate. This may partially explain the flower phenotypes of fil mutants, if during normal development interaction between abaxial and adaxial cell types is needed in the young floral primordium to induce formation of the receptacle.

FIL is related to the recently isolated CRABSCLAW $(C R C)$ gene, mutations of which lead to pleiotropic growth defects in the developing gynoecium and floral meristem (Alvarez and Smyth 1999; Bowman and Smyth 1999). CRC also shows a complex expression pattern in the developing gynoecium that includes an abaxial domain similar to but distinct from that of FIL (Bowman and Smyth 1999). Like fil, interpretations of crc mutant phenotypes may be complicated by the presence of redundant genes. Consistent with this idea is the observation that mutations in several genes have been shown to synergistically enhance the $\operatorname{crc}$ gynoecium phenotype, leading to the development of normally adaxial placentae on the abaxial surface (Bowman et al. 1999; Y. Eshed and J. Bowman, pers. comm.). These data suggest that at least one of the functions of $C R C$ is to promote abaxial cell fates during gynoecium development, although $\mathrm{crc}$ single mutants do not suggest this.

Both FIL and CRC belong to a gene family in Arabidopsis that also includes at least four other members (Bowman and Smyth 1999; Sawa et al. 1999b). All of these genes encode a helix-loop-helix/HMG-like region referred to as the YABBY domain, as well as a zinc finger domain (Bowman and Smyth 1999). The close sequence similarity shared by members of this gene family may account in part for the apparent redundancy revealed in genetic and expression studies. For example, FIL is closely related to YAB3, as these two proteins are $98 \%$ identical in the YABBY domain and $75 \%$ identical in the zinc finger region, suggesting that YAB3 might be the redundant partner to FIL (Bowman and Smyth 1999).

Another interesting member of the YABBY gene family that recently has been characterized is the INNER NO OUTER (INO) gene that is required for normal ovule development (C. Gasser, J. Villanueva, and K. Schneitz, pers. comm.). Mutations in INO result in a loss of the outer integument, a structure that normally forms on the abaxial side of the ovule primordium (Baker et al. 1997). Thus, a common theme appears to be emerging for the roles of at least three members of the YABBY class of putative transcription factors, as all appear to function to promote the abaxial identities of organs in which they are expressed.

\section{Mutants waiting to be cloned}

Other mutants that exhibit polarity phenotypes similar to those mentioned above have also been reported. leafbladeless1 (1bl1) mutants of maize have radially symmetrical leaves that are abaxialized, similar to phan mutants in snapdragon (Timmermans et al. 1998). 1 bl1 mu- tants also form leaves with patches of abaxial epidermis on the adaxial surface that produce laminar outgrowths at the ectopic adaxial-abaxial boundaries (Timmermans et al. 1998). The ectopic laminae commonly occur in pairs flanking an abaxial patch of tissue running the length of the midrib on the adaxial surface. It is proposed that the ectopic laminae are due to the juxtaposition of adaxial and abaxial territories, similar to the proposal for phan mutants. In contrast to phan mutants, all lateral organs of $1 b l 1$ plants are affected. Interestingly, $l b l 1 \mathrm{mu}-$ tants show defects in leaf founder cell recruitment, as fewer cells than in wild-type show down-regulation of a marker for nonleaf founder identity in the SAM (Timmermans et al. 1998). Although $1 b 11$ and phan mutants have many similarities, $L B L 1$ is not the maize ortholog of PHAN (Timmermans et al. 1998).

In contrast to the recessive $1 b l$ and phan mutants, the dominant phabulosa-1d (phb-1d) mutation in Arabidopsis conditions the adaxialization of leaves, and the development of axillary buds at the abaxial base of mutant leaves (McConnell and Barton 1998). phb-1d leaves are either needle-shaped and lacking in abaxial identities or trumpet shaped. The trumpet shape appears to result from a duplicated proximodistal axis with inverted adaxial-abaxial polarity forming on the abaxial base of the organ. Similar to $l b l 1$, the $p h b-1 d$ mutation affects all above ground lateral organs of the plant (McConnell and Barton 1998).

\section{Future prospects}

With the large collection of mutants and asymmetrically expressed markers now available, the future looks bright for unraveling some of the mysteries of adaxial-abaxial patterning in plants, as well as the activities of the factors already isolated. Fishing upstream of $C Y C$ and the $Y A B B Y$ genes will lead to clues specifying asymmetry in primordia, whereas going downstream of these and other genes will lead to factors that specify the growth and cell-type differences between adaxial and abaxial. Suppressor and enhancer screens should prove informative as well, as PHAN and the YABBY gene families suggest that redundant functions play a significant role in abaxial-adaxial patterning. One question raised by the uniform expression pattern of PHAN in primordia is the nature and identity of the other factors involved in creating an asymmetric effect of this putative transcription factor.

Finally, in spite of the fact that the last common ancestor of plants and animals was unicellular, remarkable similarities exist for how these organisms have evolved developmental mechanisms to pattern leaves and wings. These similarities include the early subdivision of organ (and lateral shoot) primordia into adaxial and abaxial domains of gene expression, and the juxtaposition of adaxial and abaxial domains to produce lateral growth. In spite of these striking similarities, differences between plants and animals exist, and include the absence of lineage compartments in plants (for review, see Dawe and Freeling 1991), the presence of plant cell walls that 
might limit the ability of secreted growth factors to spread, and the different developmental and life strategies of plants and animals. But then again, if leaves were wings, plants would fly.

\section{Acknowledgments}

We thank John Bowman, Ethan Bier, Detlef Weigel, and Medard $\mathrm{Ng}$ for comments on the manuscript, and John Bowman and Charles Gasser for allowing us to cite their unpublished results. A.S. is supported by the Life Sciences Research Foundation, and our Arabidopsis research is supported by grants from the $\mathrm{Na}$ tional Science Foundation and the National Institutes of Health (to M.Y.).

\section{References}

Almeida, J., M. Rocheta, and L. Galego. 1997. Genetic control of flower shape in Antirrhinum majus. Development 124: 1387-1392.

Alvarez, J. and D.R. Smyth. 1999. CRABS CLAW and SPATULA, two genes from Arabidopsis that control carpal development in parallel with AGAMOUS. Development (in press).

Baker, S.C., K. Robinson-Beers, J.M. Villanueva, J.C. Gaiser, and C.S. Gasser. 1997. Interactions among genes regulating ovule development in Arabidopsis thaliana. Genetics 145: 1109-1124.

Blair, S.S., D.L. Brower, J.B. Thomas, and M. Zavortink. 1994. The role of apterous in the control of dorsoventral compartmentalization and PS integrin gene expression in the developing wing of Drosophila. Development 120: 1805-1815.

Bowman, J.L. and D.R. Smyth. 1999. CRABS CLAW, a gene that regulates carpel and nectary development in Arabidopsis, encodes a novel protein with zinc finger and helix-loop-helix domains. Development (in press).

Bowman, J.L.. S.F. Baum, Y. Eshed, J. Putterill, and J. Alvarez. 1999. Molecular genetics of gynoecium development in Arabidopsis. Curr. Top. Dev. Biol. (in press).

Brook, W., F.J. Diaz-Benjumea and S.M. Cohen. 1996. Organizing spatial pattern in limb development. Annu. Rev. Cell Dev. Biol. 12: 61-80.

Coen, E.S. 1996. Floral symmetry. EMBO J. 15: 6777-6788.

Coen, E.S., J.M. Nugent, D. Luo, D. Bradley, P. Cubas, M. Chadwick, L. Copsy, and R. Carpenter. 1995. Evolution of floral symmetry. Phil. Trans. R. Soc. Lond. B 350: 135-38.

Cohen, B., M.E. McGuffin, C. Pfeifle, D. Segal, and S.M. Cohen. 1992. apterous: A gene required for imaginal disc development in Drosophila encodes a member of the LIM family of developmental regulatory proteins. Genes \& Dev. 6: 715-729.

Cohen, S.M. 1993. Imaginal disc development. In Drosphila Development of melanogaster (ed. M. Bate and A. MartinezArias), pp. 747-841. Cold Spring Harbor Laboratory Press, Cold Spring Harbor, NY.

Dawe, K. and M. Freeling. 1991. Cell lineage and its consequences in higher plants. Plant I. 1: 3-8.

De Celis, J.F., A. Garcia-Bellido, and S.J. Bray. 1996. Activation and function of Notch at the dorsal-ventral boundary of the wing imaginal disc. Development 122: 359-369.

Diaz-Benjumea, F.J. and S.M. Cohen. 1993. Interaction between dorsal and ventral cells in the imaginal disc directs wing development in Drosophila. Cell 75: 741-752.

- 1995. Serrate signals through Notch to establish a Wingless-dependent organizer at the dorsal/ventral compartment boundary of the Drosophila wing. Development 121: 42154225 .

Doebley, J., A. Stec, and L. Hubbard. 1997. The evolution of apical dominance in maize. Nature 386: 485-488.
Hanawa, J. 1961. Experimental studies of leaf dorsiventrality in Sesamum indicum L. Bot. Mag. Tokyo 74: 303-309.

Irvine, K. and E. Wieschaus. 1994. fringe, a boundary-specific signaling molecule mediates interactions between dorsal and ventral cells during Drosophila wing development. Cell 79: 695-606.

Kim, J., K.D. Irvine, and S.B. Carroll. 1995. Cell recognition, signal induction and symmetrical gene activation at the dorsal-ventral boundary of the developing Drosophila wing. Cell 82: 795-802.

Lawrence, P.A. and G. Struhl 1996. Morphogens, compartments, and pattern: Lessons from Drosphila? Cell 85: 951-961.

Linnaeus, C. 1749. "De peloria." Ph.D. thesis. Amoenitates Academy, Uppsala, Sweden.

Luo, D., R. Carpenter, C. Vincent, L. Copsey, and E. Coen. 1996. Origin of floral asymmetry in Antirrhinum. Nature 383: 794-799.

McConnell, J.R. and K. Barton. 1998. Leaf polarity and meristem formation in Arabidopsis. Development 125: 2935-2942.

Neumann, C.J. and S.M. Cohen. 1996. A hierarchy of crossregulation involving Notch, wingless, vestigial and cut organizes the dorsal/ventral axis of the Drosophila wing. Development 122: 3477-3485.

1997. Long-range action of Wingless organizes the dorsal-ventral axis of the Drosophila wing. Development 124: 871-880.

Rulifson, E.J. and S.S. Blair. 1995. Notch regulates wingless expression and is not required for reception of the paracrine wingless signal during wing margin neurogenesis in Drosophila. Development 121: 2813-2824.

Sawa, S., T. Ito, Y. Shimura, and K. Okada. 1999a. FILAMENTOUS FLOWER controls the formation and development of Arabidopsis infloresences and floral meristems. Plant Cell 11: 69-86.

Sawa, S., K. Watanabe, K. Goto, E. Kanaya, E.H. Morita, and K. Okada. 1999b. Molecular characterization of a meristem and organ identity gene of Arabidopsis, FILAMENTOUS FLOWER, encoding a zinc-finger and a HMG related domains. Genes \& Dev. (this issue).

Snow, M. and R. Snow. 1959. The dorsiventrality of leaf primordia. New Phytol. 58: 188-207.

Steeves, T. and I.M. Sussex. 1989. Patterns in plant development. Cambridge University Press, New York, NY.

Sussex, I.M. 1955. Morphogenesis in Solanum tuberosum L.: Experimental investigation of leaf dorsiventrality and orientation in the juvenile shoot. Phytomorphology 5: 286-300.

Timmermans, M.C.P., N.P. Schultes, J.P. Jankovsky, and T. Nelson. 1998. Leafbladeless1 is required for dorsoventrality of lateral organs in maize. Development 125: 2813-2823.

Vöchting, H. 1878-1884. Über organbildung im pflanzenreich. Physiologische untersuchungen über wachsthumsursachen und lebenseinheiten. Cohen, Bonn, Germany.

Waites, R. and A. Hudson. 1995. phantastica: A gene required for dorsoventrality in leaves of Antirrhinum majus. Development 121: 2143-2154.

Waites, R., H.R.N. Selvadurai, I.R. Oliver, and A. Hudson. 1988. The PHANTASTICA gene encodes a MYB transcription factor involved in growth and dorsoventrality of lateral organs in Antirrhinum. Cell 93: 779-789.

Wardlaw, C.W. 1949. Experiments on organogenesis in ferns. Growth (Suppl.) 9: 93-131.

Williams, J.A., S.W. Paddock, K. Vorwerk, and S.B. Caroll. 1994. Organization of wing formation and induction of a wing patterning gene at the dorsal/ventral compartment boundary. Nature 368: 299-305.

Zecca, M., K. Bassler, and G. Struhl. 1996. Direct and long-range action of a Wingless morphogen gradient. Cell 87: 833-844. 


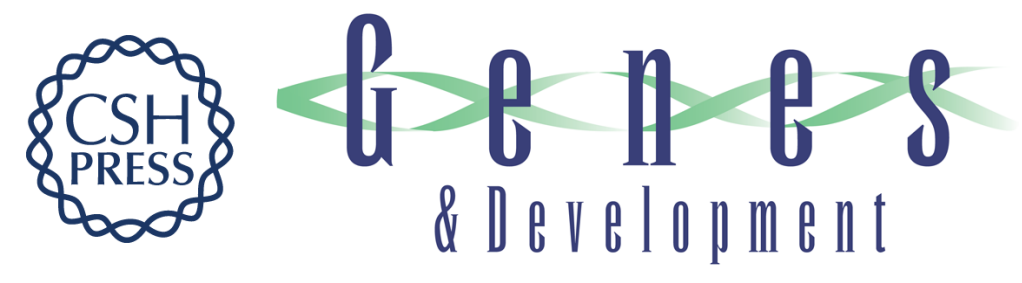

\section{Dorsoventral patterning in plants}

Allen Sessions and Martin F. Yanofsky

Genes Dev. 1999, 13:

References This article cites 27 articles, 13 of which can be accessed free at: http://genesdev.cshlp.org/content/13/9/1051.full.html\#ref-list-1

License

Email Alerting

Receive free email alerts when new articles cite this article - sign up in the box at the top Service right corner of the article or click here.

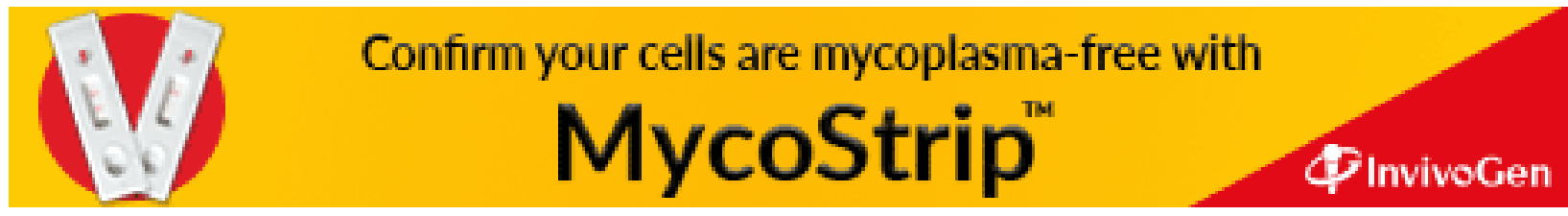

